\title{
Os fundamentos da Web Semântica como ferramentas de auxílio para as demandas da Sociedade da Informação
}

\author{
Flávia Rodrigues Elias Nunes \\ Mestre, Universidade Federal de Minas Gerais, Belo Horizonte, MG, Brasil; \\ comunicacao.flaviarodrigues@gmail.com \\ Benildes Coura Moreira dos Santos Maculan \\ Doutora, Universidade Federal de Minas Gerais, Belo Horizonte, MG, Brasil; \\ benildesmaculan@ufmg.br \\ Maurício Barcellos Almeida \\ Doutor, Universidade Federal de Minas Gerais, Belo Horizonte, MG, Brasil; \\ mba@eci.ufmg.br
}

\begin{abstract}
Resumo: As demandas da Sociedade da Informação (SI) chamam a atenção de cientistas de diversos campos de estudos sobre problemas no processo de tratamento, armazenagem, representação e recuperação da informação. Nos estudos no âmbito da Ciência da Informação (CI), conta-se com insumos de outras áreas e, nesse cenário, ganha relevância a Web Semântica, que é uma evolução do projeto inicial da web e busca oferecer, por meio das linguagens computacionais automatizadas, maior precisão na recuperação da informação. Este artigo tem como objetivo identificar os pontos de convergência entre as demandas da SI e as potencialidades da Web Semântica na resolução das problemáticas informacionais levantadas. Os principais elementos identificados (fundamentos, princípios e ferramentas) desse conceito são contextualizados em um levantamento de artigos publicados entre 2014 e 2019 na base de Dados de Artigos de Periódicos em Ciência da Informação (Brapci). Com base nesse levantamento, foi elaborado um quadro com a visão dos autores sobre alguns fundamentos e realizada uma análise com a indicação da necessidade informacional. O resultado permite concluir que a Web Semântica contribui para uma nova perspectiva no desenvolvimento de tecnologias que vêm promovendo mudanças significativas na sociedade, alterando seu comportamento, suas demandas e suas necessidades, e estão em consonância com a busca do processamento semântico das informações por meio da automatização.
\end{abstract}

Palavras-chave: Sociedade da Informação. Web Semântica. Recuperação da Informação. Refinamento Semântico. 


\title{
1 Introdução
}

As sociedades contemporâneas são marcadas pela velocidade que as mudanças acontecem. Imediatistas, elas são baseadas na informação e estão em busca de conhecimento e rapidez na transmissão de dados não lineares. Para isso, contam com a evolução das tecnologias da informação, que permitem a criação de formas mais eficazes de armazenamento, tratamento e transmissão de dados.

A quantidade e a acessibilidade às informações para os usuários são características notáveis na então chamada Sociedade da Informação. A evolução desta sociedade também modificou os valores em relação ao acesso à informação, à organização, à mediação e ao uso dela e do conhecimento, justificando o investimento em pesquisas que visam amenizar a problemática existente (VALENTIM, 2013).

O universo online tem sido um dos principais focos de atenção, já que a internet vem se consolidando como um campo fértil para a disseminação e armazenamento de dados. A World Wide Web, que surgiu no início da década de 1990, foi uma evolução da proposta inicial da internet, que é de ser um sistema de distribuição de comunicação entre computadores. $\mathrm{O}$ projeto implantou $\mathrm{o}$ conceito de hipertexto, oferecendo, assim, interfaces acessíveis para os usuários, conforme pode ser visto na declaração do britânico Timothy John Berners-Lee, criador da World Wide Web.

\begin{abstract}
A invenção da World Wide Web envolveu uma crescente percepção de que havia grande poder em se arrumar as ideias de uma maneira não restritiva, como em uma teia. E essa percepção foi surgindo precisamente através desse tipo de processo. A Web surgiu como resposta a um desafio em aberto, através de um redemoinho de influências, ideias e realizações de muitos lados até que, por extraordinários ofícios da mente humana, um novo conceito se materializou. Foi um processo de crescimento por etapas, e não uma solução linear de um problema bem definido após o outro (BERNERS-LEE, 1999, p. 3).
\end{abstract}

Alinhado ao desenvolvimento da web está a origem das redes sociais que, na atual SI, faz com que fique ainda mais em evidência o fato de que o internauta seja um participante ativo na produção de conteúdo. $\mathrm{O}$ ambiente digital possibilita que o usuário se expresse e seja ouvido. Ou seja, a comunicação passa a ser bilateral, deixando de ser um privilégio de um só 
emissor, e a diversidade, o grande volume de dados e a dinâmica dos sistemas levam a grandes desafios para organizar, processar e analisar os dados gerados.

A web evoluiu e se estabeleceu um novo estágio que ganhou espaço nos estudos de recuperação da informação, descrita por alguns autores como Web Semântica. Por meio da criação de padrões, da arquitetura de metadados, de serviços de inferências e ontologias, esse conceito vai além do fato de apenas os seres humanos processarem as informações. A esfera da Web Semântica é dotada de ferramentas que permitem que as máquinas, na forma de agentes computacionais, identifiquem conceitos e processem as informações e os possíveis sentidos evocados pelos relacionamentos entre conceitos dos dados disponíveis na internet. De acordo com Berners-Lee, Hender e Lassila (2001, p. 2, tradução nossa), “a Web Semântica é uma extensão da web atual, onde a informação possui um significado claro e bem definido, possibilitando uma melhor interação entre computadores e pessoas". Diante disso, este artigo tem como objetivo identificar os pontos de convergência entre as demandas da SI e as potencialidades da Web Semântica na resolução de problemáticas informacionais atuais. Para alcançar tal propósito, foi realizada uma análise onde foi apresentado um breve panorama das atuais necessidades da sociedade contemporânea. Foi enfatizada a importância de investigações com insumos de outras disciplinas, assim como aquelas que envolvam o estudo do sentido e o desenvolvimento de aplicações tecnológicas, com vistas ao avanço das pesquisas no campo da CI. Foram também expostos alguns dos principais elementos da Web Semântica, por meio da coleta de 16 artigos selecionados sobre o assunto na Base de Dados de Artigos de Periódicos em Ciência da Informação (Brapci). Por fim, foi elaborado um quadro que identificou e relacionou os pontos de convergência entre as demandas apresentadas e os fundamentos da Web Semântica apontadas de acordo com visão dos autores dos artigos coletados.

Para tanto, depois desta introdução, a seção 2 apresenta o conceito de SI, apontando quais são as exigências dos indivíduos na atual conjuntura; na seção 3 discorre-se sobre os fundamentos, princípios e ferramentas da Web Semântica, 
trazendo, na seção 4, um levantamento da literatura que traz insumos para corroborar tais elementos; por fim, a seção 5 apresenta as considerações finais.

\section{A Sociedade da Informação e suas demandas na contemporaneidade}

O termo "Sociedade da Informação" é creditado por vários autores aos japoneses Yujiro Hayashi e Jiro Kamishima. O primeiro publicou o livro "The Information Society: from hard to soft" e Jiro Kamishima, em 1963, lançou, em um artigo, ideias sobre a SI. Foi a partir daí que o tema ganhou grande repercussão no Japão. Castells (1999), por sua vez, atribui a Nora e Minc (1980) esse termo e destaca que a nomenclatura define a sociedade baseada na economia informacional, enfatizando que "o termo informacional indica o atributo de uma forma específica de organização social em que a geração, o processamento e a transmissão da informação tornam-se as fontes fundamentais de produtividade e poder devido às novas condições tecnológicas surgidas nesse período histórico" (CASTELLS, 1999, p. 64-65).

Com a sociedade denominada de SI, alguns autores salientam a importância de considerar o conhecimento e os fatores que sustentam o desenvolvimento humano como fundamentais da evolução da sociedade (ESTEVES, 2000). Burch (2005), por exemplo, diz que a UNESCO defende o uso do termo "Sociedade do Conhecimento", uma vez que, neste caso, não apenas leva-se em consideração o avanço tecnológico, mas também transformações sociais, culturais, econômicas, políticas e institucionais, dando, assim, uma perspectiva mais pluralista a esse desenvolvimento.

Unger e Freire (2008) declaram que Bell (1973), Masuda (1982), Castells (1999) e Webster (1995) destacam a forte presença da tecnologia no advento da SI. Freitas (2002a) corrobora e diz que o resultado do momento evolutivo é caracterizado pelo uso de recursos e tecnologias informacionais e pela ampliação dessas ferramentas no processamento, estocagem e transmissão da informação, com maior evidência no processamento delas em variadas atividades que são permeadas pela convergência de tecnologias de telecomunicação. Seja em uma visão micro, da informação como um registro do conhecimento, ou a visão macro, que é o próprio conhecimento acumulado na 
sociedade contemporânea, fato é que a tecnologia trouxe grandes transformações. Castells (1999) afirma que a habilidade das sociedades em dominar as tecnologias da informação e comunicação é fundamental para a transformação delas e cita que elas são, atualmente, o que as "novas fontes de energia foram para as revoluções industriais sucessivas, do motor a vapor à eletricidade, aos combustíveis fósseis e até mesmo à energia nuclear" (CASTELLS, 1999, p. 68).

Ao mesmo tempo em que tais avanços trouxeram vantagens, pode-se afirmar que vieram incertezas, cujas consequências são difíceis de prever. Ramalho e Ouchi (2011, p. 66) assinalam que o grande volume de informações disponível é apresentado como desafio contemporâneo, uma vez que torna-se necessária a "singularização contextual na reconstrução do conhecimento, possibilitando a contextualização das informações a partir de requisitos de pertinência e relevância", auxiliando na recuperação de interesse do usuário.

A produção informacional em grande escala vem acompanhada do fato de que o usuário passou a interagir e a criar conteúdo, tornando-se fonte de informação. Segundo Almeida (2001), mais do que ter acesso à tecnologia, uma das características dessa sociedade é a utilização desses mecanismos para a busca e seleção de informações, de forma a resolver problemas do cotidiano. Castells (1999), da mesma forma que Almeida, destaca a aplicabilidade das tecnologias em uma estrutura organizada.

\footnotetext{
O que caracteriza a atual revolução tecnológica não é a centralidade de conhecimentos e informação, mas a aplicação desses conhecimentos e dessa informação para a geração de conhecimentos e de dispositivos de processamento/comunicação da informação, em um ciclo de realimentação cumulativo entre a inovação e seu uso. [...]. O ciclo de realimentação entre a introdução de uma nova tecnologia, seus usos e seus desenvolvimentos em novos domínios torna-se muito mais rápido no novo paradigma tecnológico (CASTELLS, 1999, p. 69).
}

Esteves (2000) chama a atenção para o fato de que vivemos em um meio tecnologizado e estritamente ligado às características do capitalismo, trazendo grandes mudanças nos serviços da informação, além de mudanças econômicas, ao permitirem, com agilidade, acesso a dados e informações de diversas partes, motivando uma readequação social (PEREIRA; SILVA; GIRON, 2014). 
De acordo com Pezzotti (2019), um estudo da União Internacional das Telecomunicações, órgão vinculado à Organização das Nações Unidas (ONU), o número de internautas já é de cerca 3.9 bilhões de pessoas. Já o relatório Global Digital Report 2018, de janeiro de 2018, revela que o Brasil tinha cerca de 130 milhões de usuários ativos em redes sociais online, correspondendo a cerca de 55\% da população brasileira se considerarmos a estimativa de 209 milhões de pessoas no país, segundo o Instituto Brasileiro de Geografia e Estatística (IBGE). Esses dados mostram o rápido progresso tecnológico e apontam quais os processos estão se tornando obsoletos na rápida evolução da economia digital e indicam melhores segmentos de investimento.

Para unir a produção de conteúdo informal e a interatividade proposta pelo "bate-papo" e as mensagens instantâneas, nasceram as redes sociais, que são sistemas que exigem a construção de uma persona por meio de um perfil ou página pessoal e permite a interação com o público.

Redes Sociais são dinâmicas e estão sempre em transformação. Essas transformações, em uma rede social, são largamente influenciadas pelas interações. É possível que existam interações que visem somar a construir um determinado laço social e interações que visem enfraquecer ou mesmo destruir outro laço (RECUERO, 2009, p. 78).

Neste contexto de espaço interativo, as pessoas estão cada vez mais conectadas e em busca de novas fontes para suprir suas necessidades de informações. Isso não só aumentou significativamente a produção de conteúdo, como permitiu que os usuários se transformassem em emissores de informação e em construtores da notícia em tempo real. Na SI, o uso da informação tornou-se, então, peça fundamental para que o usuário se tornasse um agente ativo dentro da rede, que produz novos conteúdos e "gera coletivos inteligentes que podem alimentar o ciclo informacional: informação - conhecimento - desenvolvimento - informação" (BARRETO, 1998, p. 123).

O crescimento da utilização do ambiente online para a criação e difusão das informações trouxe consigo problemas quanto à confiança na veracidade dos dados e na recuperação assertiva por parte do usuário. Surge, nesse contexto, fenômenos como o da pós-verdade, um tema recente que vem ganhando mais notoriedade devido ao grande número de informações falsas que são 
disseminadas nas plataformas digitais e em diferentes mídias sociais, como Instagram, Facebook, Twitter, Youtube e WhatsApp.

O termo "pós-verdade", inclusive, foi considerada a palavra do ano de 2016 pelo Dicionário Oxford, que a definiu como aquilo "que se relaciona ou denota circunstâncias nas quais fatos objetivos têm menos influência em moldar a opinião pública do que apelos à emoção e a crenças pessoais" (OXFORD DICTIONARIES, 2016, online). Esse fato acaba gerando o efeito contrário, que é o da desinformação que, conforme indicado por Cunha e Cavalcanti (2008, p. 120), se refere à "escassez de informação, informação errônea". No inglês, há dois termos para representar esse conceito, misinformation e disinformation, sendo que a primeira se refere a informações incorretas ou falsas que são propagadas intencionalmente, mas sem perceber que elas são inverdades. Já a segunda diz respeito às informações imprecisas ou falsas propagadas deliberadamente com a intenção de enganar.

A desinformação já vem sendo tratada desde os anos 1980 por Fox (1983). Em seu trabalho pioneiro, o autor sustenta que a informação não necessariamente tem de ser verdadeira para ser considerada informativa. Sobre essa questão, Zattar (2017) considera que as desinformações devem ser evitadas pelos profissionais da informação, trazendo elementos importantes para a SI, que são a observação da qualidade das fontes e da veracidade das informações.

Além da escassez de informação ou informação errônea, outros tipos de desinformação são a dissimulação, a ambivalência, a distorção e a falsificação, conforme apontam Zhou e Zhang (2007). No ciberespaço, Monteiro (2006) destaca que há centro de significância estruturado, hierarquizado, linear ou instrumentos de organização do conhecimento que reproduzam o modelo de significância, sentido único e referência fixa.

A convergência de opiniões destacadas pelos autores citados sobre as atuais mudanças na sociedade contemporânea, que é baseada nas potencialidades da informação e na crescente evolução tecnológica, demonstra, de maneira impactante, os desafios do Cientista da Informação em atender às demandas informacionais atuais. Após essas considerações, foram elencadas, 
sinteticamente, no Quadro 1, algumas das principais necessidades identificadas da SI.

Quadro 1 - Demandas da Sociedade da Informação

1 - Produção constante de conteúdo e disseminação imediata das informações no ambiente online.

2 - Desenvolvimento de sistemas automatizados que registrem o conhecimento e a memória intelectual, de forma que os conteúdos produzidos não se percam em meio à grande produção informacional.

3 - Para tornar a informação utilizável é preciso ampliação da tecnologia midiática que permite maior acessibilidade ao usuário.

4 - A crescente produção e convergência de dados e de plataformas informacionais na web provoca urgente necessidade da gestão dos processos de representação, armazenamento e recuperação da informação.

5 - Refinamento nos modelos de representação da informação na máquina, com ferramentas semanticamente refinadas, evitando os possíveis problemas de ambiguidade.

6 - Evolução nas ferramentas e parâmetros de busca online, permitindo uma recuperação mais assertiva pelo usuário.

7 - Com o aumento da interatividade na internet, em especial nas redes sociais online, o usuário passa a ser um agente ativo na produção de conteúdo e disseminação das informações, resultando na necessidade da verificação da veracidade das informações, evitando assim a proliferação de desinformações e pós-verdades.

Fonte: elaborado pelos autores (2019).

Diante das características da SI e o crescimento constante da produção informacional na web, torna-se cada vez mais necessária a contribuição de outros campos de estudo para a busca de soluções aos desafios da contemporaneidade. Como já mencionado, o desenvolvimento das tecnologias da informação foi responsável também pelo início de uma nova era, marcada por mudanças comportamentais, sociais, econômicas, políticas e corporativas, além de pesquisas que buscam em diversas áreas de interface insumos para o desenvolvimento das pesquisas em CI. Sobre esse momento, é possível ainda aplicar o que Polizelli (2008) se refere como: 
tecnologias de informática e comunicações (TIC) para cooperação e compartilhamento de conhecimento entre atores, a fim de disseminar a formação de competências na população (POLIZELLI, 2008, p. 2).

Essa característica do diálogo entre diversos campos de estudo já era defendida por Saracevic (1996), que afirmou que a CI é interdisciplinar por natureza. Ele ressalta, contudo, que essas relações são dinâmicas e podem ser mais fortes em um determinado tempo e espaço com algumas áreas científicas. Quanto à ligação com a tecnologia de informação, o autor afirma que isso é algo inexorável, já que o componente tecnológico está impulsionando e forçando a evolução da CI, assim como a evolução da Sociedade da Informação. Hjorland (2000) também reforça que a troca de conhecimentos entre disciplinas é sinal de uma ciência em progresso. Rojas, Souza e Cintra (2008), por sua vez, complementa que o diálogo entre disciplinas surge pela complexidade do fenômeno a ser estudado. Ele afirma ainda que é indispensável que cada disciplina possua identidade própria para poder interagir com as demais.

No ambiente da web, Alvarenga (2001) destaca a necessidade de estudos que envolvam mais de uma área e que possibilitem o desenvolvimento de novas tecnologias:

O desafio de organizar a Internet extrapola hoje fronteiras disciplinares e torna-se a busca de uma solução para que realmente o valor do meio possa efetivamente ser usufruído em toda a sua potencialidade. Dentre os profissionais que se atêm a trabalhar nessa área destacam-se filósofos, linguistas, profissionais da ciência da computação, da psicologia cognitiva, da linguística e da semiologia, todos focalizando as dificuldades atuais e procurando "uma luz no fundo do túnel" (ALVARENGA, 2001, p. 10)

Alves e Barbosa (2010) também corroboram sobre a importância de estudos e pesquisas provenientes de outras áreas. Segundo os autores, o compartilhamento da informação e do conhecimento faz parte de um vasto campo de estudo para pesquisadores de diferentes áreas, como administração, CI, psicologia, comunicação e tecnologia da informação. E, assim como outras áreas, a CI possui função social e humana, sendo ativa na evolução da sociedade da informação.

Nesse sentido, para o aprofundamento da compreensão dos processos de recuperação da informação da atual sociedade no meio digital, faz-se necessário 
os estudos multidisciplinares que visam contribuir para uma otimização semântica dos sistemas.

\section{Fundamentos, princípios e ferramentas da Web Semântica}

Como exposto anteriormente, a Sociedade da Informação tem como demanda constante os estudos multidisciplinares que visem alcançar melhorias nos processos de tratamento e recuperação da informação, com o desenvolvimento de mecanismos que permitam organizar e adicionar informações semânticas aos dados. Com as tecnologias da informação e da comunicação, houve um grande avanço não só na quantidade de informações produzidas, quanto na criação de formatos mais adequados dos modelos multidisciplinares de organização e recuperação.

A web vem acompanhando constantemente essas modificações, em um processo natural de agregação de significado e, devido à necessidade de ter uma compreensão melhor das informações, que a princípio eram incompreensíveis para os mecanismos computacionais, desenvolveu-se os princípios da Web Semântica. Com a produção informacional em larga escala, a qualidade e relevância da informação ganharam ainda mais importância, sendo requisitos essenciais no procedimento de classificação da infinidade de dados que devem ser organizados, identificados e recuperados pelo usuário no contexto que realmente necessita. Essa classificação, em âmbito do raciocínio humano já é compreendida, mas o modo que as informações são interpretadas pelas máquinas e disponibilizadas para os usuários é outro desafio.

A concepção da Web Semântica tem evoluído, sendo considerada uma boa opção para o desenvolvimento de mecanismos que viabilizam a recuperação da informação e o fornecimento de bases para a criação de aplicações mais inteligentes, que se baseiam na maior capacidade do software em interpretar os conteúdos em rede, possibilitando uma indexação mais eficaz, promovendo a compreensão da informação pelas máquinas. Ou seja, além dos dados serem capazes de serem buscados pelos usuários, as máquinas estariam aptas a compreendê-los, proporcionando o retorno assertivo em determinado contexto. 
Bozsak et al. (2002) reflete sobre essa questão ao dizer que a Web Semântica se esforçou, no sentido de construir uma nova arquitetura na web em que o conteúdo tem uma semântica formal, possibilitando um entendimento dos agentes automatizados. Acredita-se que a CI mostra-se relevante no contexto do raciocínio de máquina, uma vez que junto às tecnologias colabora, por meio do desenvolvimento de ferramentas de busca e base de dados semântica, para uma melhor integração do vocabulário controlado à estrutura da Web, conforme é destacado por Paletta e Mucheroni (2014).

A Web Semântica é uma Web de Dados - de dados, títulos, identificações, propriedades e quaisquer outros dados que se possa produzir. As tecnologias da Web Semântica [...] fornecem um ambiente onde uma aplicação pode consultar esses dados e/ou fazer inferências usando vocabulários controlados (PALETTA; MUCHERONI, 2014, p. 80).

Freitas (2002b) explica que na Web Semântica a representação dos dados é feita por meio de uma linguagem que descreve o conteúdo semântico e os significados contextuais, além da estrutura e da forma de exibição de documentos. Para que ela se tornasse implementável e a recuperação viável na Web, uma série de ferramentas de outras áreas de conhecimento tem sido amplamente utilizada nos processos que favorecem o entendimento da informação pela máquina, além dos serviços de interoperabilidade e cooperação no ambiente digital. Entre essas linguagens de programação estão o XML (eXtensible Markup Language), o RDF (Resource Description Framework), o RDF Scheme, o OWL (Web Ontology Language), o SPARQL e outros agentes computacionais que são descritos pelo Word Wide Web Consortium (W3C), que reúne empresas, instituições acadêmicas, profissionais e cientistas com o objetivo principal de desenvolver estudos e tecnologias que possibilitem potencializar as funcionalidades da Web.

A primeira linguagem computacional desenvolvida com características da Web Semântica foi a eXtensible Markup Language (XML), que tem como objetivo criar uma infraestrutura única, em formato textual e semanticamente estruturada. Berners-Lee, Hender e Lassila (2001) fizeram toda a proposição da arquitetura da Web Semântica baseada no XML, visando gerar um padrão de 
assimilação na troca de documentos eletrônicos, de forma textual, simples, estruturada, extensível, flexível, semanticamente rica e com uma segurança adequada para diversos tipos de linguagens. Assim, a linguagem XML permitiu e permite a criação de estruturas concisas nos documentos contidos na Web, tornando-os processáveis por máquinas.

Sobre o XML, Oliveira (2002) explica que:

Ao contrário da HTML, que através das marcas pré-definidas gerenciam os textos marcados e controlam sua representação estabelecendo ligações entre os documentos, a linguagem XML marca semanticamente um documento. XML consiste em padrão utilizado para marcação de documentos que contém informações estruturadas, ou seja, documentos que contêm uma estrutura clara e precisa da informação armazenada e obtida com XML. Esta estruturação define e separa claramente conteúdo, significado e apresentação. Assim os documentos em XML podem ser indexados com maior precisão que as páginas planas escritas em HTML (OLIVEIRA, 2002, p. 5).

Para a implementação da linguagem XML, destacam-se na arquitetura da Web Semântica o Unicode e a URI. O padrão de codificação Unicode é um conjunto de caracteres únicos, originalmente baseado no alfabeto inglês, que suporta todos os idiomas, tendo sido criados em diferentes tipos para desenvolver as tecnologias no ambiente Web, uma vez que define uma correlação entre símbolos e números. Já a URI (Uniform Resource Identifier) é uma sequência de caracteres (string) que identifica, de maneira individual e inequívoca, cada recurso (imagem, página, entre outros) da Web.

$\mathrm{Na}$ arquitetura da Web Semântica, destaca-se também o Resource Description Framework (RDF), uma linguagem computacional em formato de sentença que se tornou um padrão recomendado pelo $\mathrm{W} 3 \mathrm{C}$ e tem como objetivo o enriquecimento semântico. Souza (2004) afirma que a ideia do RDF é a descrição dos dados e dos metadados por meio de um esquema de "triplas" (sujeito, predicado e objeto), que é feita pela combinação de um recurso, uma propriedade e um valor para a propriedade de um recurso. Já o RDF Schema (RDFS), uma extensão do RDF, é usado para a criação de vocabulários básicos com noções de classe, hierarquia e relações, possibilitando assim um maior nível de abstração, definição de conceitos e tipos de objetos. 
No nível do vocabulário ontológico, como é o caso da linguagem Web Ontology Language (OWL), são definidas um grande número de relações, permitindo caminhos de escrita lógica formal. Almeida (2007) confirma isso e explica que a operacionalização em uma linguagem única é possível uma vez que a ontologia "possui conceitos, relações e atributos semanticamente bem definidos e pode variar em grau de formalidade, conforme a necessidade" (ALMEIDA, 2007, p. 78). Com a utilização delas é possível definir classes com a união ou intersecção de outras. De acordo com Ramalho Vidotti e Fujita (2005), nessa camada está, atualmente, a maioria das pesquisas relacionadas à Web Semântica.

A utilização de ontologias favorece o compartilhamento da mesma estrutura de informações entre pessoas e softwares agentes e permite a reutilização do conhecimento em um domínio. Uma página na web pode ser relacionada automaticamente com outras de uma rede de conhecimento. De acordo com Jacob (2003):

[...] na Web Semântica, uma ontologia é uma conceitualização parcial de um dado domínio do conhecimento, compartilhado por uma comunidade de usuários, que tem sido definido em uma linguagem formal e processável por máquina para a proposta explícita de compartilhar informações semânticas de dados através de sistemas automatizados (JACOB, 2003, p. 19).

Além disso, considerando que as ontologias são linguagens artificiais, cujo objetivo é deixar os termos organizados de forma mais compreensível pelo usuário, Teixeira e Almeida (2019) destacam ainda que é possível “enxergar as ontologias como instrumentos de organização da informação capazes de reduzir a ambiguidade" (TEIXEIRA; ALMEIDA, 2019, p. 12).

O SPARQL, por sua vez, é linguagem que estrutura consulta semântica para RDF, que na Web Semântica tem como principal função a recuperação da informação por meio de queries, que extraem informações de fontes de dados baseado em triplas. Para Santarém Segundo (2014, p. 3870-3871), “[...] o SPARQL é um conjunto de especificações que fornecem linguagens e protocolos para consultar e manipular o conteúdo publicado em RDF na Web".

Visando correlacionar as demandas da SI, sintetizadas no Quadro 1, com os elementos (fundamentos, princípios e ferramentas) da Web Semântica, 
buscou-se na literatura os insumos que poderiam contribuir na busca de soluções para as sete problemáticas apontadas, conforme descrito na próxima seção.

\section{Procedimentos metodológicos e análise}

Para o levantamento na literatura foi utilizado como fonte de coleta a Base de Dados de Artigos de Periódicos em Ciência da Informação (Brapci), uma base referencial brasileira, produto do projeto de pesquisa "Opções metodológicas em pesquisa: a contribuição da área da informação para a produção de saberes no ensino superior", de Bufrem et al. (2010). A base de dados online possui indexados artigos publicados nas revistas científicas e profissionais da CI desde 1972.

Para a composição do corpus, a busca teve como critério a presença do termo "Web Semântica" no título, resumo e palavras-chave em artigos publicados entre 2014 a 15 de março de 2019. Como resultado, foram recuperados na base do Brapci um total de 21 artigos. Nem todos foram utilizados na análise, uma vez verificado que alguns tinham a mesma autoria e, basicamente, os mesmos fundamentos eram apontados. O corpus final foi composto por 16 artigos e o resultado da análise está exposto no Quadro 2, que apresenta, por meio da percepção dos autores dos artigos encontrados, os elementos (fundamentos, princípios e ferramentas) da Web Semântica.

\begin{tabular}{|c|c|c|}
\hline Autor & Fundamentos da Web Semântica & $\begin{array}{c}\text { Correlação: } \\
\text { demandas Quadro } 1\end{array}$ \\
\hline $\begin{array}{l}\text { Souza, } \\
\text { Almeida, Porto } \\
\text { (2013) }\end{array}$ & $\begin{array}{l}\text { A semântica formal não é capaz de lidar com a } \\
\text { linguagem natural utilizada com o propósito da } \\
\text { comunicação. Ou seja, a linguagem da Web } \\
\text { Semântica ainda é restrita a algumas sentenças } \\
\text { similares à linguagem natural, mas que são } \\
\text { adequadas para máquinas. }\end{array}$ & Item 4 e 5 \\
\hline Catarino (2014) & $\begin{array}{l}\text { Os Simple Knowledge Organization System } \\
\text { (SKOS), ou sistemas de organização do } \\
\text { conhecimento, escritos na linguagem computacional } \\
\text { RDF, que é uma linguagem computacional } \\
\text { recomendada pelo W3C, torna os esquemas de } \\
\text { conceitos expressos passíveis de serem lidos por } \\
\text { agentes inteligentes. }\end{array}$ & Item 2 \\
\hline Lopes (2014) & $\begin{array}{l}\text { A identificação de URI nos repositórios Linked } \\
\text { Open Data dá aos agentes computacionais uma } \\
\text { ferramenta para reduzir ou eliminar a ambiguidade } \\
\text { na expressão da informação. }\end{array}$ & Item 5 \\
\hline
\end{tabular}


Os fundamentos da Web Semântica como ferramentas auxílio para as demandas da Sociedade da Informação Flávia Rodrigues Elias Nunes, Benildes Coura Moreira dos Santos Maculan e Maurício Barcellos Almeida

\begin{tabular}{|c|c|c|}
\hline Autor & Fundamentos da Web Semântica & $\begin{array}{c}\text { Correlação: } \\
\text { demandas Quadro } 1\end{array}$ \\
\hline \multirow{2}{*}{ Santos (2014) } & $\begin{array}{l}\text { A implantação da web semântica contribui para a } \\
\text { integração da indexação, dos recursos tecnológicos } \\
\text { e das atividades de recuperação da informação que } \\
\text { resultam em recursos mais sofisticados e que } \\
\text { otimiza os serviços de acesso e uso da informação. }\end{array}$ & Item 6 \\
\hline & $\begin{array}{l}\text { Os recursos básicos da web semântica são } \\
\text { articulados nas camadas de tecnologia da web e } \\
\text { padrões. As camadas superiores são Logic (escrita } \\
\text { de regras), Proof (execução das regras) e Trust } \\
\text { (avaliação em conjunto com a camada de confiança } \\
\text { para camada de confiança para aplicações e dados). }\end{array}$ & Item 7 \\
\hline $\begin{array}{l}\text { Catarino, } \\
\text { Cervantes, } \\
\text { Andrade (2015) }\end{array}$ & $\begin{array}{l}\text { O projeto da Web Semântica tem como valor social } \\
\text { o compartilhamento do conhecimento em escala } \\
\text { global, em que todos possam ter acesso ao conteúdo } \\
\text { da Web, independente de tipo de hardware, } \\
\text { software, infraestrutura de rede, idioma, cultura, } \\
\text { localização geográfica ou capacidade física e } \\
\text { mental. }\end{array}$ & Item 1 e 3 \\
\hline $\begin{array}{l}\text { Marsulo, } \\
\text { Carvalho, } \\
\text { Santarém } \\
\text { Segundo } \\
(2015)\end{array}$ & $\begin{array}{l}\text { A noção de ontologia é uma forma de } \\
\text { estabelecimento de limites na elaboração conceitual } \\
\text { e na construção da arquitetura da Web Semântica, } \\
\text { fato que permite que a máquina possa "inferir" } \\
\text { conhecimento novo a partir de conhecimento } \\
\text { existente, atendendo assim a necessidades dos } \\
\text { grupos de usuários. }\end{array}$ & Item 5 e 6 \\
\hline \multirow[b]{2}{*}{$\begin{array}{l}\text { Nakano et al. } \\
(2015)\end{array}$} & $\begin{array}{l}\text { As URIs evitam ambiguidades para o entendimento } \\
\text { das máquinas. }\end{array}$ & Item 5 \\
\hline & $\begin{array}{l}\text { A Web Semântica exige esforços interdisciplinares } \\
\text { para alcançar seu potencial, como da Ciência da } \\
\text { Computação, Inteligência Artificial, Ciência da } \\
\text { Informação e Design da Informação. }\end{array}$ & Item 1,2 e 3 \\
\hline $\begin{array}{l}\text { Nhacuongue, } \\
\text { Dutra (2016) }\end{array}$ & $\begin{array}{l}\text { A Web Semântica, no contexto da organização do } \\
\text { conhecimento, contribui para a disponibilização, } \\
\text { acesso, o uso e o reuso dos dados, explorando as } \\
\text { potencialidades dos computadores. }\end{array}$ & Item 1 e 3 \\
\hline $\begin{array}{l}\text { Santarém } \\
\text { Segundo, } \\
\text { Coneglian } \\
(2016)\end{array}$ & $\begin{array}{l}\text { A linguagem XML tem como característica a } \\
\text { facilidade em interoperar dados, o RDF tem a } \\
\text { capacidade de conectar recursos, já as ontologias } \\
\text { possibilitam tornar computável a conceitualização } \\
\text { de domínios e processos, o SPARQL interage } \\
\text { diretamente com os dados e torna possível a } \\
\text { recuperação da informação. }\end{array}$ & Item 5 \\
\hline $\begin{array}{l}\text { Aquarone, Luz } \\
(2017)\end{array}$ & $\begin{array}{l}\text { A Web Semântica, em especial as ontologias, } \\
\text { podem contribuir para que a localização e a seleção } \\
\text { das informações relevantes possam atender às } \\
\text { necessidades dos usuários de forma personalizada. }\end{array}$ & Item 6 \\
\hline $\begin{array}{l}\text { Arakaki, } \\
\text { Simionato, } \\
\text { Santos (2017) }\end{array}$ & $\begin{array}{l}\text { As diversas linguagens computacionais } \\
\text { desenvolvidas a partir dos estudos da Web } \\
\text { Semântica, como o Protocol and RDF Query } \\
\text { Language (SPARQL), proporcionam consultas } \\
\text { semânticas em conjuntos de dados. }\end{array}$ & Item 2 e 6 \\
\hline
\end{tabular}


Os fundamentos da Web Semântica como ferramentas auxílio para as demandas da Sociedade da Informação Flávia Rodrigues Elias Nunes, Benildes Coura Moreira dos Santos Maculan e Maurício Barcellos Almeida

\begin{tabular}{|l|l|l|}
\hline \multicolumn{1}{|c|}{ Autor } & \multicolumn{1}{|c|}{ Fundamentos da Web Semântica } & \multicolumn{1}{c|}{ Correlação: } \\
demandas Quadro 1
\end{tabular}

Fonte: Quadro elaborado pelos autores (2019).

Percebe-se que os fundamentos da Web Semântica estão em consonância com as demandas da SI, a começar com uma das suas premissas, que é tornar os dados mais acessíveis a todos os tipos de usuários e, para isso, é necessário contar com as aplicações tecnológicas. Como visto, alguns autores citados, como Catarino, Cervantes, Andrade (2015) e Nhacuongue e Dutra (2016), 
confirmam que a Web Semântica é uma ferramenta que contribui para essa disponibilização dos dados. Nakano et al. (2015) complementam e apontam que, para a Web Semântica alcançar o seu potencial, é necessário a contribuição de insumos de outros campos de estudos.

Outra situação identificada é que em meio à grande massa de informações gerada, inclusive pelos próprios indivíduos que passaram a interagir e produzir conteúdo, e o surgimento de diversas mídias e contextos novos, fez com que a web se transformasse. Além da acessibilidade das informações e dos sistemas automatizados para armazenamento dos dados, outra demanda da SI é a necessidade da evolução de gestão dos processos de representação e recuperação das informações. Luz, Coneglian, Santarém Segundo (2019), por exemplo, citam o Linked Data, como uma possível aplicação da Web Semântica para essa gestão. Ainda nessa linha da recuperação mais assertiva, as linguagens de programação, como XML, RDF, RDF Schema, Ontologias e SPARQL, também contribuem para os desafios contemporâneos, facilitando o processamento das informações pelas máquinas.

Pelo levantamento teórico foi percebido que o uso de metadados e tags é fundamental para os requisitos de pertinência e relevância, promovendo uma recuperação direcionada e satisfatória. Ou seja, como apontado por Coneglian et al. (2019), é possível fazer uma contextualização dos dados, deixando a busca mais personalizada.

Além disso, os recursos dinâmicos da Web Semântica contribuem para o enriquecimento conceitual da representação, resultando em uma melhoria na usabilidade e acessibilidade, além de diminuição das ambiguidades. Monteiro (2018) enfatiza que a tecnologia é uma grande aliada, reproduzindo a capacidade interpretativa que os agentes computacionais têm. Ou seja, a Web Semântica preza pelo significado do conteúdo, facilita o gerenciamento da informação e dá aos agentes computacionais uma forma semelhante à do homem para o entendimento dos sentidos e conexões com diferentes fontes de dados.

A questão da confiabilidade das informações, por outro lado, necessita de processos em que os agentes saibam identificar e validar a autenticidade das fontes e a confiança nas informações. Santos (2014) explica as camadas de 
tecnologia da web que auxiliam na arquitetura básica da web semântica proposta pela W3W e explicita com uma imagem de Koivunen e Miller (2001, p. 34).

Figura 1 - Camadas da Web Semântica

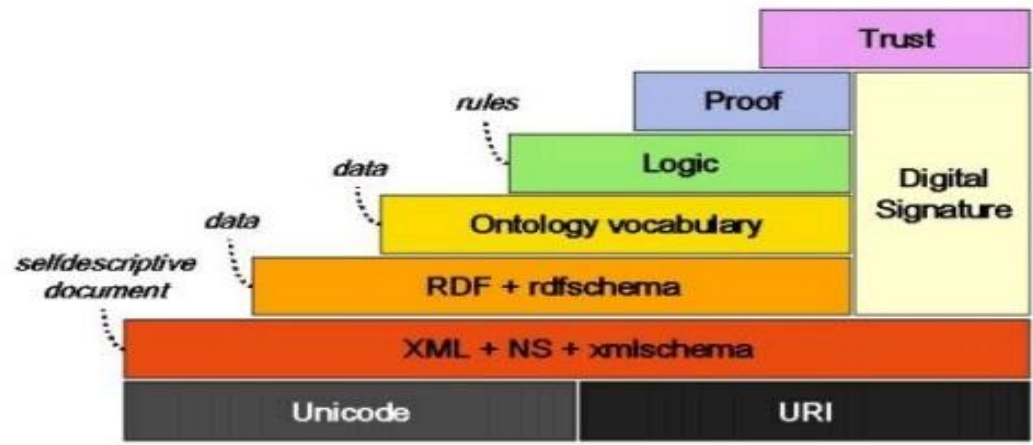

Fonte: Koivunen \& Miller (2001).

Os autores explicam que os objetivos das camadas superiores Logic, Proof e Trust são escritas de regras; execução das regras e avaliação em conjunto com a camada de confiança para aplicações e dados.

Buscando complementar a análise e indicar de forma mais assertiva os pontos de convergência buscados, o Quadro 3 exibe de forma sintetizada essa correlação. Para isso, as sete necessidades indicadas no Quadro 1 foram agrupadas na primeira coluna em três serviços da informação (Representação e Gestão, Recuperação e Verificação) e, na segunda coluna, estão as conclusões da análise sobre a contribuição da Web Semântica para tais serviços.

Quadro 3 - Os Serviços da Informação e a Web Semântica

\begin{tabular}{|l|l|}
\hline Serviços da Informação & \multicolumn{1}{|c|}{ Contribuição da Web Semântica } \\
\hline \multirow{2}{*}{ Representação e Gestão da Informação } & $\begin{array}{l}\text { Por meio de padrões de formatação de dados, } \\
\text { como o XML, RDF, RDF Schema, Ontologias } \\
\text { e SPARQL, a Web Semântica tornou-se um } \\
\text { movimento colaborativo, que visa organizar e } \\
\text { representar a informação de linguagem natural } \\
\text { em uma maneira legível para a máquina. } \\
\text { Esses padrões atribuem significados ao a } \\
\text { conteúdo online, colaborando na } \\
\text { desambiguição dos esquemas de conceitos } \\
\text { representados. }\end{array}$ \\
\hline $\begin{array}{l}\text { As características de indexação e } \\
\text { representação da informação contribuem para } \\
\text { a atribuição de significado ao conteúdo. Isso }\end{array}$ \\
\hline
\end{tabular}




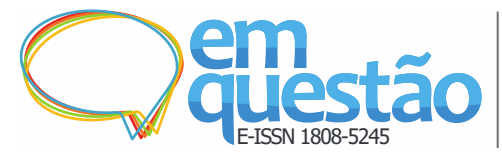

Os fundamentos da Web Semântica como ferramentas auxílio para as demandas da Sociedade da Informação Flávia Rodrigues Elias Nunes, Benildes Coura Moreira dos Santos Maculan e Maurício Barcellos Almeida

\begin{tabular}{|l|l|}
\hline Recuperação da Informação & $\begin{array}{l}\text { se dá por meio de ferramentas e tecnologias } \\
\text { que realizam anotações semânticas, resultando } \\
\text { em uma recuperação mais assertiva. Ou seja, } \\
\text { o usuário consegue fazer uma pergunta mais } \\
\text { elaborada a partir de várias relações, ao invés } \\
\text { de buscar por palavras isoladas. }\end{array}$ \\
\hline Verificação do conteúdo & $\begin{array}{l}\text { A Web Semântica vai além da ideia de } \\
\text { acessibilidade e encriptação de dados. Sua } \\
\text { camada mais alta, chamada de Trust, atua } \\
\text { sobre a capacidade de inferência e } \\
\text { confiabilidade do conteúdo, possibilitando a } \\
\text { verificação da legitimidade da informação. }\end{array}$ \\
\hline
\end{tabular}

Fonte: Quadro elaborado pelos autores (2019).

\section{Considerações finais}

Este artigo procurou abordar a relevância da revolução tecnológica na transformação do comportamento da atual sociedade contemporânea, conhecida como Sociedade da Informação. Essa alteração ocorreu no campo não só comportamental, como social, organizacional, econômico, político, entre outros. A grande produção informacional, que ganhou ainda mais força com o ambiente online, alterou significativamente a relação do homem com a informação e os meios de armazenagem, tratamento, representação e recuperação da informação, ganhando a atenção de profissionais de diferentes áreas de estudo, já que o surgimento progressivo de dados ocorreu em todos os níveis.

De acordo com os autores citados, com a evolução do projeto da Web, o tratamento das informações ganhou ainda mais relevância, já que o usuário não é mais apenas participante passivo, mas peça-chave na construção e disseminação de conteúdo. O interesse em pesquisas acerca do assunto ganhou a atenção de profissionais de diversos campos de atuação, que contribuem para a evolução de ferramentas semânticas. E é inegável o fato de que tecnologias desenvolvidas no âmbito da web têm resultado em uma série de avanços e melhorias significativas para a representação de recursos informacionais.

Diante do panorama exposto sobre os fundamentos da Web Semântica e as necessidades da atual SI, pode-se concluir que a Web Semântica tem sido temática de estudo para a CI, principalmente no que tange aos procedimentos ligados às ciências documentais, como pesquisa, indexação e representação eletrônica dos documentos, armazenamento e recuperação da informação. As 
linguagens aqui citadas, como XML, RDF, assim como as ontologias, são interfaces da área da Ciência da Computação, e trazem não só os benefícios operacionais mencionados, mas também para o usuário final, que aproveita da tecnologia e dos estudos semânticos aplicados nessas ferramentas para ter acesso às informações qualificadas e de modo mais rápido.

\section{Agradecimentos}

Agradecemos ao apoio recebido do Conselho Nacional de Desenvolvimento Científico e Tecnológico $(\mathrm{CNPq})$. O terceiro autor agradece o apoio do Conselho Nacional de Desenvolvimento Científico e Tecnológico, Ministério da Educação - SHIS QI 1 Conjunto B, Blocos A, B, C e D - Lago Sul, Brasília / DF - CEP 71605-001. Processo 303050/2016-0 CNPq nº 12/2016.

\section{Referências}

ALMEIDA, Maria Elizabeth Biancocini de. Tecnologia na Escola: criação de redes de conhecimento. Série "Tecnologia na Escola" - Programa Salto para o Futuro, 2001.

ALMEIDA, Maurício Barcellos. Aplicação de Ontologias em Segurança da Informação. Fonte, Belo Horizonte, v. 4, n. 7, p. 75-83, 2007.

ALVARENGA, Lídia. A Teoria do Conceito Revisitada em Conexão com Ontologias e Metadados no Contexto das Bibliotecas Tradicionais e Digitais. DataDramaZero:Revista de Informação, v. 2, n. 6, dez. 2001.

ALVES, Alessandra; BARBOSA, Ricardo Rodrigues. Influências e barreiras ao compartilhamento da informação: uma perspectiva teórica. Ciência da Informação, Brasília, v. 39, n. 2, p. 115-128, 2010.

AQUARONE, Débora Cristina Bonfim; LUZ, Charlley dos Santos. A conectividade e a organização da informação: uma abordagem entre a internet das coisas e a web semântica. Ciência da Informação em Revista, Maceió, v. 4, n. 2, p. 24-38, 2017.

ARAKAKI, Felipe Augusto; SIMIONATO, Ana Carolina; SANTOS, Plácida Leopoldina Ventura Amorim da Costa. Catalogação e tecnologia: interseções com a web semântica. Informação@Profissões, Londrina, v. 6, n. 2, p. 3-19, 2017. DOI: $10.5433 / 2317-4390.2017 v 6 n 2 p 03$

BARRETO, Aldo de Albuquerque. Mudança estrutural no fluxo do conhecimento: a comunicação eletrônica. Ciência da Informação, Brasília, v. 27, n. 2, p. 122-127, maio/ago. 1998. 
Os fundamentos da Web Semântica como ferramentas auxílio para as demandas da Sociedade da Informação Flávia Rodrigues Elias Nunes, Benildes Coura Moreira dos Santos Maculan e Maurício Barcellos Almeida

BELL, Daniel. O advento da sociedade pós-industrial: uma tentativa de previsão social. São Paulo: Cultrix, 1973.

BERNERS-LEE, Tim. Weaving the Web: The Past, Present and Future of the World Wide Web by its Inventor. London: Orion Business Books, 1999.

BERNERS-LEE, Tim; HENDER, James; LASSILA, Ora. The Semantic Web: a new form of Web content that is meaningful to computers will unleash a revolution of new possibilities. Scientifc American, New York, v. 284, n. 5, Maio 2011.

BOZSAK, Erol. et al. KAON: towards a large scale Semantic Web. In: ECOMMERCE AND WEB TECHNOLOGIES, 3., 2002, Aix-en-Provence. Proceedings... Springer, Lecture Notes in Computer Science. v. 2455. Disponível em: http://www.aifb.unikarlsruhe.de/WBS/dob/pubs/ecWeb2002.pdf._Acesso em: 8 mar. 2019.

BUFREM, Leilah Santiago et al. Modelizando práticas para a socialização de informações: a construção de saberes no ensino superior. Perspectivas em Ciência da Informação, Belo Horizonte, v. 15, n. 2, p. 22-41, 2010.

BURCH, Sally. Sociedade da informação/sociedade do conhecimento. In: AMBROSI, Alain; PEUGEOT, Valérie; PIMIENTA, Daniel. Desafios das palavras. Caen: C\&F Éditions, 2005.

CASTELLS, Manuel. A sociedade em rede. 8. ed. rev. e ampl. São Paulo: Paz e Terra, 1999.

CATARINO, Maria Elisabete; CERVANTES, Brígida Maria Nogueira; ANDRADE, Ilza Almeida de. A representação temática no contexto da web semântica. Informação \& Sociedade: Estudos, João Pessoa, v. 25, n. 3, p. 105$116,2015$.

CATARINO, Maria Elisabete. Simple knowledge organization system: construindo sistemas de organização do conhecimento no contexto da web semântica. Informação \& Tecnologia, João Pessoa, v. 1, n. 1, p. 17-28, 2014.

CONEGLIAN, Caio Saraiva et al. Tecnologias da Web Semântica na arquitetura da informação. Revista Interamericana de Bibliotecología, Medellín, v. 42, n. 1, p. 23-35, 2019.

CUNHA, Murilo Bastos; CAVALCANTI, Cordélia Robalinho de Oliveira. Dicionário de Biblioteconomia e Arquivologia. Brasília: Briquet de Lemos, 2008.

ESTEVES, João Pissara. A Sociedade de Informação: tecnologia, sound bites ou a transparência remitificada. Revista.txt - Textos de Cultura e Comunicação, n. $42,2000$. 
Os fundamentos da Web Semântica como ferramentas auxílio para as demandas da Sociedade da Informação Flávia Rodrigues Elias Nunes, Benildes Coura Moreira dos Santos Maculan e Maurício Barcellos Almeida

FOX, Christopher John. Information and misinformation: an investigation of the notions of information, misinformation, informing, and misinforming. Westport, CT: Greenwood, 1983.

FREITAS, Frederico Luiz Gonçalves. Sistemas multiagentes cognitivos para a recuperação, classificação e extração integradas de informação da Web. 2002. Tese (Doutorado em Engenharia Elétrica) - Universidade Federal de Santa Catarina, Florianópolis, $2002 b$.

FREITAS, Lídia Silva de. A memória polêmica da noção de Sociedade da Informação e sua relação com a área de informação. Informação \& Sociedade: Estudos, João Pessoa, v. 12, n. 2, p. 1-23, 2002 a.

GLOBAL DIGITAL REPORT 2018. We Are Social and Hootsuite. Essential insights into internet, social media, mobile and ecommerce use around the world. Disponível em: https://digitalreport.wearesocial.com. Acesso em: 8 mar. 2019.

HJØRLAND, Birger. Documents, memory institutions and information science. Journal of Documentation, v. 56, n. 1, p. 27-41, 2000.

JACOB, Elin K. Ontologies and the Semantic Web. Bulletin of the American Society for Information Science and Technology, v. 9, n. 4, p. 18-22, Apr./May 2003.

JORENTE, Maria José Vicentini; PADUA, Mariana Cantisani; SANTAREM SEGUNDO, José Eduardo. Criação de padrões na web semântica: perspectivas e desafios. Em Questão, Porto Alegre, v. 23, n. 3, p. 157-178, 2017.

KOIVUNEN,Marja-Riitta ; MILLER Eric. W3C Semantic Web Activity. In Semantic Web Kick-Off in Finland: Vision, Technologies, Research, and Applications, Finlândia, 2001.

LOPES, Antônio Tavares. Um esquema XSD genérico para expressão de Normas MARC: princípios de conceção orientada para a Web Semântica. Páginas A\&B, Arquivos e Bibliotecas, Portugal, n. 2, p. 96-107, 2014.

LUZ, Charlley dos Santos; CONEGLIAN, Caio Saraiva; SANTAREM SEGUNDO, José Eduardo. Tecnologias da web semântica para a recuperação da informação no wikidata. Revista Digital de Biblioteconomia \& Ciência da Informação, Campinas, v. 17, p. 1-20, 2019.

MARSULO, Thabyta Giraldelli; CARVALHO, Ângela Maria Grossi de; SANTAREM SEGUNDO, José Eduardo. Bibliotecas digitais: um campo de aplicação web semântica. Revista Prisma.com, Aveiro, n. 29, p. 139-156, 2015. 
Os fundamentos da Web Semântica como ferramentas auxílio para as demandas da Sociedade da Informação Flávia Rodrigues Elias Nunes, Benildes Coura Moreira dos Santos Maculan e Maurício Barcellos Almeida

MASUDA, Yoneji. A sociedade da informação como sociedade pósindustrial. Rio de Janeiro: Editora Rio, 1982.

MONTEIRO, Silvana Drumond. O ciberespaço e os mecanismos de busca: novas máquinas semióticas. Ciência da Informação, Brasília, v. 1, n. 1, p. 3138, jan./abr. 2006.

MONTEIRO, Silvana Drumond. A vida secreta dos metadados no wikidata: um enfoque sobre o sentido na (web) semântica formal. Informação \& Sociedade: Estudos, João Pessoa, v. 28, n. 1, p. 181-190, 2018.

NAKANO, Natália et al. Web semântica no repositório brapci: uma revisão da literatura. Brazilian Journal of Information Science, Marília, v. 9, n. 2, 2015.

NHACUONGUE, Januário Albino; DUTRA, Moisés Lima. De Paul Otlet à Web Semântica: aportes teóricos sobre a organização do conhecimento. Informação \& Tecnologia, João Pessoa, v. 3, n. 1, p. 138-158, 2016.

NORA, Simon.; MINC, Alain. A informatização da sociedade. Rio de Janeiro: FGV, 1980.

OLIVEIRA, Rosa Maria Vivona Bertonlini. Web Semântica: novo desafio para os profissionais da informação. In: SEMINÁRIO NACIONAL DE BIBLIOTECAS UNIVERSITÁRIAS, 12., 2002, Recife. Anais [...]. Recife: UFPE, 2002.

OXFORD DICTIONARIES. Word of the year 2016 is.... Oxford, [s. d.]. Disponível em: https://en.oxforddictionaries.com/word-of-the-year/word-of-theyear-2016. Acesso em: 20 mar. 2019.

PALETTA, Francisco Carlos; MUCHERONI, Marcos Luiz. O desenvolvimento da Web 3.0: Linked Data e DBPEDIA. Prisma.com, Porto, n. 25, p. 7390,2014

PEREIRA, Agostinho Oli Koppe; SILVA, Fabiana Barcelos da; GIRON, Jerônimo. Sociedade tecnológica: a informação e o consumo como reflexos da modernidade. Revista Novos Estudos Jurídicos, Itajaí, v. 19, n. 1, jan./abr. 2014.

PEZZOTTI, Renato. Com 3,9 bilhões de usuários no mundo, o que acontece na web em um minuto?. Disponível em:

https://economia.uol.com.br/noticias/redacao/2019/04/01/com-39-bilhoes-deusuarios-no-mundo-o-que-acontece-na-web-em-um-minuto.htm. Acesso em: 09 abr. 2020.

POLIZELLI, Luiz Demerval. Sociedade da Informação: iniciando o debate. In: POLIZELLI, Luiz Demerval.; OZAKI, Adalton. (org.). Sociedade da Informação: os desafios da era da colaboração e da gestão do conhecimento. São Paulo: Saraiva, 2008. 
Os fundamentos da Web Semântica como ferramentas auxílio para as demandas da Sociedade da Informação Flávia Rodrigues Elias Nunes, Benildes Coura Moreira dos Santos Maculan e Maurício Barcellos Almeida

RAMALHO, Rogério Aparecido Sá; OUCHI, Marcos Teruo. Tecnologias semânticas: novas perspectivas para representação de recursos informacionais. Informação \& Informação, Londrina, v. 16, n. 3, p. 60-75, jan./jun. 2011.

RAMALHO, Rogério Aparecido Sá; VIDOTTI, Silvana Aparecida Borsetti Gregorio; FUJITA, Mariângela Spotti Lopes. Web Semântica: aspectos interdisciplinares para a organização e recuperação de informações. In: ENCONTRO NACIONAL DE PESQUISA EM CIÊNCIA DA INFORMAÇÃO, 6., 2005, Florianópolis. Anais [...]. Florianópolis: UFSC, 2005.

RECUERO, Raquel. Redes Sociais na Internet. Porto Alegre: Sulinas, 2009.

ROJAS, Jucimara; SOUZA, Regina Aparecida Marques de; CINTRA, Rosana Carla. Dinâmica do trabalho e a organização do Espaço na Educação Infantil. Cuiabá: Ed. UFMT, 2008.

SANTAREM SEGUNDO, José Eduardo; CONEGLIAN, Caio Saraiva. Web semântica e ontologias: um estudo sobre construção de axiomas e uso de inferências. Informação \& Informação, Londrina, v. 21, n. 2, p. 217-244, 2016.

SANTAREM SEGUNDO, José Eduado. Web Semântica: introdução a recuperação de dados usando Sparql. In: ENCONTRO NACIONAL DE PESQUISA EM CIÊNCIA DA INFORMAÇÃO, 15, Belo Horizonte, MG, 2014. Anais eletrônicos... Belo Horizonte, MG: ANCIB, 2014. Disponível em <http://enancib2014.eci.ufmg.br/documentos/anais/anais-gt8>

SANTOS, Thaís Helen do Nascimento. Entre o tecnológico e a complexidade: aplicações da web semântica nos sistemas de informação de arquivos. Revista Prisma.com, Aveiro, n. 22, p. 108-130, 2014.

SARACEVIC, Tefko. Ciência da informação: origem, evolução e relações. Perspectivas em Ciência da Informação, Belo Horizonte, v. 1, n. 1, p. 41-62, 1996.

SOUZA, Renato Rocha; ALMEIDA, Maurício Barcellos; PORTO, Renata Maria Abrantes Baracho. Ciência da informação em transformação: big data, nuvens, redes sociais e web semântica. Ciência da Informação, Brasília, v. 42, n. 2, 2013.

SOUZA, Renato Rocha; ALVARENGA, Lídia. A Web Semântica e suas contribuições para a ciência da informação. Ciência da Informação, Brasília, v. 33, n.1, p. 132-141, jan./abr. 2004.

TEIXEIRA, Lívia Maragon Duffles; ALMEIDA, Maurício Barcellos. Aspectos ontológicos e epistêmicos em terminologias clínicas: em busca de 
Os fundamentos da Web Semântica como ferramentas auxílio para as demandas da Sociedade da Informação Flávia Rodrigues Elias Nunes, Benildes Coura Moreira dos Santos Maculan e Maurício Barcellos Almeida

interoperabilidade semântica no ambiente médico. Status: no prelo, Encontros Bibli, Florianópolis, v. 23, n. 53, p. 1-15, 2019.

UNGER, Roberto José Gervásio; FREIRE, Isa Maria. Regimes de Informação na Sociedade de Informação: uma contribuição para a gestão da Informação.

Revista Digital de Biblioteconomia e Ciência da Informação, Campinas, v. 2, n. 2, p. 87-114, jan./jun. 2008.

VALENTIM, Marta Lígia Pomim (org.). Ambientes e fluxos de informação em contextos empresariais: o setor cárnico de Salamanca/Espanha. Brazil Journal of Information Science, Marília, v. 7, n. Especial, p. 299-323, 2013.

WEBSTER, Frank. Information and the idea of an information society. In: WEBSTER, Frank. Theories of the information society. London: Routtedge, 1995. Cap. 2, p. 6-51.

ZAIDAN, Fernando Hadad et al. Wittgenstein e o significado dos nomes na web semântica. Ciência da Informação, Brasília, v. 47, n. 3, 2018.

ZATTAR, Marianna. Competência em informação e desinformação: critérios de avaliação do conteúdo das fontes de informação. Liinc em Revista, Rio de Janeiro, v. 13, n. 2, p. 285-293, nov. 2017.

ZHOU, Lina; ZHANG, Dongsong. An ontology-supported misinformation model: toward a digital misinformation library. IEEE Transactions on Systems, Man, and Cybernetics--Part A: Systems and Humans, v. 37, n. 5, p. 804-813, 2007.

\title{
The fundaments of Web Semantics as helping tools for the demands of Information Society
}

\begin{abstract}
Demands of the Information Society (IS) are a real concern for scientists from several fields with respect to issues of information organization, storage, representation e retrieval. Information Science studies (IS) use resources of other fields, and, in this setting, "Web Semantics", which is an evolution of the initial web project, becomes relevant, and it aims at offering, by means of automatized computational languages, higher precision in the retrieval of information. This paper's goal is to identify the convergence points amongst IS demands and the potentialities of Web Semantics in the solving of informational problems found. The concept's main elements identified (foundations, principles and tools) are contextualized in a survey published between 2014 and 2019 in the Base de Dados de Artigos de Periódicos em Ciência da Informação (Brapci). Based on this survey, a board with the authors'
\end{abstract}


view on some foundations and an analysis with indication for informational needs have been developed. The results lead to the conclusion that Web Semantics contributes for a new perspective in the developing of new technologies which have been promoting significant changes in society, altering its behavior, demands and needs, and are in consonance with the search for semantic processing of information by means of automatization.

Keywords: Information Society. Web Semantics. Information Recovering. Semantic Refinement.

Recebido: 29/04/2019

Aceito: 11/10/2019 